\title{
Stationary localized states due to nonlinear impurities described by the modified discrete nonlinear Schrödinger equation
}

\author{
Bikash C. Gupta and Sang Bub Lee \\ Department of Physics, Kyungpook National University, Taegu 702-701, Korea
}

\begin{abstract}
The modified discrete nonlinear Schrödinger equation is used to study the formation of stationary localized states in a one-dimensional lattice with a single impurity and an asymmetric dimer impurity. A periodically modulated and a perfectly nonlinear chain is also considered. Phase diagrams of localized states for all systems are presented. From the mean square displacement calculation, it is found that all states are not localized even though the system comprises random nonlinear site energies. Stability of the states is discussed.
\end{abstract}

PACS number : 71.55.-i,72.10.Fk

\section{INTRODUCTION}

Localized states appear due to the presence of impurity or disorder (which breaks the translational symmetry) in the system [1]. While there have been studies on the formation of localized states due to linear impurities in various systems [1], only a few look into the formation of localized states due to nonlinear impurities. The discrete nonlinear Schrödinger equation [2-16] employed to study the formation of stationary localized (SL) states is given by

$$
i \frac{d C_{n}}{d t}=\epsilon_{n} C_{n}+V\left(C_{n+1}+C_{n-1}\right)-\chi_{n}\left|C_{n}\right|^{\sigma} C_{n},
$$

where $\sigma$ is a constant, $C_{n}$ is the probability amplitude of the particle (exciton) to be at the site $n, \epsilon_{n}$ and $\chi_{n}$ are, respectively, the static site-energy and the nonlinear strength at site $n$, and $V$ is the nearest neighbor hopping element. The nonlinear term $\left|C_{n}\right|{ }^{\sigma} C_{n}$ arises due to the interaction of the exciton with the lattice vibration 15,16. If the oscillators are purely harmonic in nature, $\sigma=2$. The Eq. (1), has been used to study the formation of SL states in a one-dimensional chain as well as in the Cayley tree with single and dimer nonlinear impurities [3 6,10 12]. For the case of a perfectly nonlinear chain where $\chi_{n}=\chi$ for all $n$ it has been shown that SL states are possible even though the translational symmetry of the system is preserved [8, 9, 12 14]. These results were remarkably different from those of the corresponding systems with linear impurities. The Eq. (1) has been derived with the assumption that the lattice oscillators in the system are local and their motions are much faster than the exciton motion. A natural question to be raised is what happens to the formation of SL states when the oscillators in the lattice are harmonic in nature and coupled with their nearest neighbors. In such system, in the presence of the interaction of the exciton with the lattice vibration, the time evolution equation governing the dynamics of the exciton has been derived by Kopidakis et al. [17] as

$$
i \frac{d C_{n}}{d t}=\epsilon_{n} C_{n}+V\left(C_{n+1}+C_{n-1}\right)
$$

$$
-\chi_{n}\left(\left|C_{n+1}\right|^{2}+\left|C_{n-1}\right|^{2}+2\left|C_{n}\right|^{2}\right) C_{n},
$$

where $C_{n}, \epsilon_{n}, V$ and $\chi_{n}$ carries the same meanings as in Eq. (1). The Eq. (2) is called the 'modified' discrete nonlinear Schrödinger equation. The Hamiltonian which generates Eq. (2) is given by

$$
\begin{aligned}
H & =\sum_{n=-\infty}^{\infty}\left(C_{n}^{\star} C_{n+1}+C_{n} C_{n+1}^{\star}\right) \\
& +\sum_{n=-\infty}^{\infty} \frac{\chi_{n}}{2}\left(\left|C_{n+1}\right|^{2}+\left|C_{n-1}\right|^{2}+2\left|C_{n}\right|^{2}\right)\left|C_{n}\right|^{2} .
\end{aligned}
$$

We note that the Eq. (2) has more nonlinear terms compared to the Eq. (1) and, in addition, the Eq. (2) is more relevant in condensed matter physics. To the best of our knowledge, this equation has not been used to study the formation of SL states even though an equation similar to Eq. (2) has been used to study the formation SL states in one-dimensional system due to a single impurity and a symmetric dimer impurity only [18]. Therefore, it is essential to consider the right equation (i.e., Eq. (2)) for the elaborate study of the formation of SL states in one-dimensional systems. Thus we study it here.

This paper is organized as follows. In Sec. II, we discuss the effect of a single impurity (i.e., $\chi_{n}=\chi \delta_{n, 0}$ ) on the formation of SL states. In Sec. III we consider the case of a dimer impurity, $\chi_{n}=\left(\chi_{1} \delta_{n, 0}+\chi_{2} \delta_{n, 1}\right)$. In Sec. IV the periodically modulated nonlinear chain as well as a perfectly nonlinear chain will be considered. In Sec. V, we consider the presence of random nonlinearities through out the lattice. The stability of the states are discussed in Sec. VI. Finally we summarize our findings in Sec. VII.

\section{SINGLE NONLINEAR IMPURITY}

Consider the system of a one-dimensional chain with a nonlinear impurity at the cener site. The time evolution of an exciton in the system is governed by Eq. (2) with $\chi_{n}=\chi \delta_{n, 0}$. The Hamiltonian which produces the 
equation of motion for the exciton in the system is given by

$$
\begin{aligned}
H & =\sum_{n}\left(C_{n}^{\star} C_{n+1}+C_{n} C_{n+1}^{\star}\right) \\
& -\frac{\chi}{2}\left(\left|C_{1}\right|^{2}+\left|C_{-1}\right|^{2}+2\left|C_{0}\right|^{2}\right)\left|C_{0}\right|^{2} .
\end{aligned}
$$

Since $\sum_{n}\left|C_{n}\right|^{2}$ is a constant of motion, we suitably renormalize so that $\sum_{n}\left|C_{n}\right|^{2}=1$. Therefore, $\left|C_{n}\right|^{2}$ can be considered as the probability for the exciton to be at site $n$. Since we are interested in the stationary localized states, we consider the ansatz

$$
C_{n}=\phi_{n} \exp (-i E t) ; \quad \phi_{n}=\phi_{0} \eta^{|n|}
$$

where $\eta$ lies between 0 and 1 and can be asymptotically defined as $\eta=\frac{|E|-\sqrt{E^{2}-4}}{2}, E$ being the energy of the localized state which appears outside the host band. Since in a one-dimensional system, states appearing outside the band at the expanse of the states in the continuum are exponentially localized, the ansatz in Eq. (5) is justified and may also be readily derived from the Green function analysis [10,11]. Substituting the ansatz to the normalization condition we get

$$
\left|\phi_{0}\right|^{2}=\frac{1-\eta^{2}}{1+\eta^{2}} .
$$

Direct substitution of $\phi_{0}, \phi_{n}$ and hence $C_{n}$ in terms of $\eta$ in Eq. (4) yields an effective Hamiltonian,

$$
H_{\mathrm{eff}}=\frac{4 \eta}{1+\eta^{2}}-\frac{\chi\left(1-\eta^{2}\right)^{2}}{\left(1+\eta^{2}\right)} .
$$

The fixed point solutions of the reduced dynamical system described by $H_{\text {eff }}$ will give the values of $\eta$ which correspond to the localized state solutions 9]. It should be noted that since $\chi$ is constant, the effective Hamiltonian is a function of only one dynamical variable $\eta$. Thus fixed point solutions are readily obtained from the condition $\partial H_{\mathrm{eff}} / \partial \eta=0$, i.e.,

$$
\frac{2}{|\chi|}=\eta\left(3+\eta^{2}\right)=f(\eta)
$$

Thus, for a fixed value of $\chi$, the number of solutions of Eq. (8) lying between 0 and 1 will correspond to the number possible SL states. We note that $f(\eta)$ is a monotonically increasing function of $\eta$ and will be maximum at $\eta=1$. Thus, we see that there is no solution for $|\chi|<0.5$ and only one solution for $|\chi| \geq 0.5$. Therefore, we conclude that there is a critical value of $|\chi|=0.5$ separating the no-state region from the one-state region. On the other hand, for the same system described by Eq. (1), the critical value of $|\chi|$ separating the no-state region from the one-state region is 2 . Thus the nearest neighbor coupling of the lattice oscillators are responsible to reduce the critical value of nonlinear strength by a factor of four. Therefore, we expect that next nearest neighbor coupling of the lattice oscillators will further reduce the critical value of the nonlinear strength and thus the critical value may approach to zero when the oscillators are globally coupled.

\section{DIMER NONLINEAR IMPURITY}

We consider a one-dimensional lattice with two nonlinear impurities placed at site 0 and 1 denoted by $\chi_{n}=$ $\left(\chi_{1} \delta_{n, 0}+\chi_{2} \delta_{n, 1}\right)$. If $\chi_{1}=\chi_{2}$, we call it symmetric-dimer impurity and otherwise asymmetric-dimer impurity. We will here consider the system with asymmetric-dimer impurity, since the results for the symmetric-dimer impurity will be obtained as a special case. The one-dimensional chain with the asymmetric dimer impurity can be described by Eq. (3) with $\chi_{n}=\left(\chi_{1} \delta_{n, 0}+\chi_{2} \delta_{n, 1}\right)$. For stationarity condition we assume that $C_{n}=\phi_{n} \exp (-i E t)$. Furthermore, for localized states we assume the following forms for $\phi_{n}$.

$$
\begin{array}{ll}
\phi_{n}=[\operatorname{sgn}(E) \eta]^{n-1} \phi_{1} ; & n \geq 1, \\
\phi_{-|n|}=[\operatorname{sgn}(E) \eta]^{|n|} \phi_{0} ; & n \leq 0,
\end{array}
$$

with $\eta$ as defined earlier. The ansatz given by Eq. (9) is exact and can be derived from the Green function analysis. It is also justified from the fact that the states which appear outside the host band are exponentially localized [10]. Three different cases are possible: (i) $\phi_{1}=\phi_{0}$ (symmetric), (ii) $\phi_{1}=-\phi_{0}$ (antisymmetric) and (iii) $\phi_{1} \neq \phi_{0}$ (asymmetric). To consider all three cases, we introduce a variable $\beta=\frac{\phi_{0}}{\phi_{1}}$. Without any loss of generality, $\phi_{0}$ and $\phi_{1}$ can be assumed to be real. Therefore, the value of $\beta$ is confined between 1 and -1 if $\left|\phi_{0}\right| \leq\left|\phi_{1}\right|$. Else we inverse the definition of $\beta$. Thus, $\beta=1$ corresponds to the symmetric states, $\beta=-1$ corresponds to the antisymmetric states, and $-1 \leq \beta \leq 1$ corresponds to the asymmetric states. Substituting the ansatz in Eq. (9) and the definition of $\beta$ in the normalization condition, $\sum_{n}\left|C_{n}\right|^{2}=1$, we obtain

$$
\left|\phi_{0}\right|^{2}=\frac{1-\eta^{2}}{1+\beta^{2}}
$$

Using the ansatz and the normalization condition, we obtain the effective Hamiltonian as a function of $\beta$ and $\eta$, given as

$$
\begin{aligned}
H_{\mathrm{eff}} & =2 \beta \frac{1-\eta^{2}}{1+\beta^{2}}+2 \operatorname{sgn}(E) \eta \\
& -\frac{\chi_{1}}{2}\left(\frac{\left(1-\eta^{2}\right)^{2}}{1+\beta^{2}}+\frac{\left(1+\eta^{2}\right)\left(1-\eta^{2}\right)^{2}}{\left(1+\beta^{2}\right)^{2}}\right) \\
& -\frac{\chi_{2}}{2}\left(\frac{\left(\beta^{2}+\beta^{4} \eta^{2}+2 \beta^{4}\right)\left(1-\eta^{2}\right)^{2}}{\left(1+\beta^{2}\right)^{2}}\right) .
\end{aligned}
$$

If $\beta= \pm 1$, we obtain 


$$
\begin{aligned}
H_{\mathrm{eff}}^{ \pm}= & \pm\left(1-\eta^{2}\right)+2 \operatorname{sgn}(E) \eta \\
& -\frac{\chi_{1}}{2}\left(\frac{\left(1-\eta^{2}\right)^{2}}{2}+\frac{\left(1+\eta^{2}\right)(1-\eta)^{2}}{4}\right) \\
& -\frac{\chi_{2}}{2}\left(\frac{\left(3+\eta^{2}\right)\left(1-\eta^{2}\right)^{2}}{4}\right)
\end{aligned}
$$

where ' + ' corresponds to the symmetric states and '-' corresponds to the antisymmetric states. The number of fixed point solutions of the reduced dynamical system described by $H_{\text {eff }}$ gives the possible number of SL states. The fixed point solutions satisfy the equation,

$$
\begin{aligned}
& 4(\operatorname{sgn}(E) \mp \eta) \\
& +\chi_{1}\left(2 \eta\left(1-\eta^{2}\right)-\frac{\eta\left(1-\eta^{2}\right)^{2}}{2}+\eta\left(1-\eta^{4}\right)\right) \\
& +\chi_{2}\left(\eta\left(1-\eta^{2}\right)\left(3+\eta^{2}\right)-\frac{\eta\left(1-\eta^{2}\right)^{2}}{2}\right)=0 .
\end{aligned}
$$

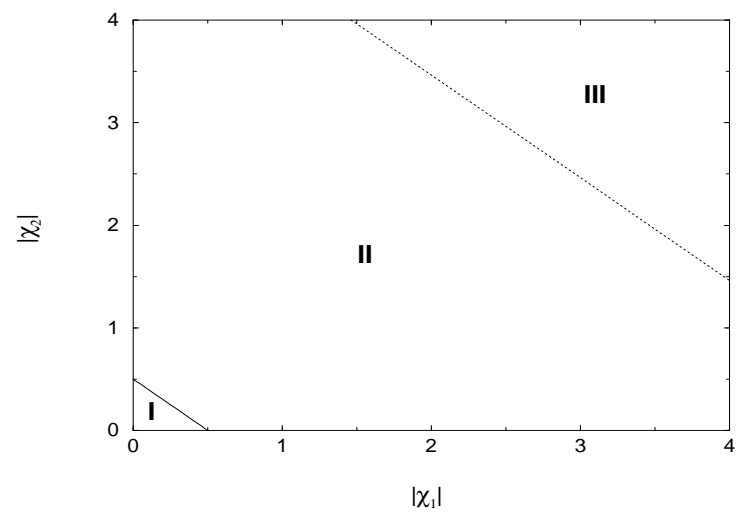

FIG. 1. Phase diagram of symmetric and antisymmetric SL states for the one-dimensional chain with a dimer impurity. Region I has no SL state, region II has one symmetric SL state and region III has one symmetric SL state and two antisymmetric SL states.

After solving this equation for each point in the $\left(\left|\chi_{1}\right|,\left|\chi_{2}\right|\right)$ plane, we obtain the number of possible symmetric as well as antisymmetric SL states in the $\left(\left|\chi_{1}\right|,\left|\chi_{2}\right|\right)$ plane. The phase diagram is shown in Fig. 1. The solid line is the critical line separating the no-symmetric-state region from the one symmetricstate-region and, the dotted line is the critical line separating no-antisymmetric-state region from the twoantisymmetric-states region. Thus, there is no state in region I, one symmetric state in region II, and one symmetric and two antisymmetric states in region III. Furthermore, there is one symmetric state on the solid and, one symmetric state and one antisymmetric state on the the dotted line.

Now, let us find the asymmetric states where $\beta \neq 1$. The effective Hamiltonian is a function of two dynamical variables, $\beta$ and $\eta$. Therefore the fixed point solutions will obey the equations given by

$$
\frac{\partial H_{\mathrm{eff}}}{\partial \eta}=0 \text { and } \frac{\partial H_{\mathrm{eff}}}{\partial \beta}=0
$$

After a little algebra we obtain the desired equations,

$$
\begin{aligned}
& 2\left(1-\beta^{4}\right)+\chi_{1} \beta\left(1-\eta^{2}\right)\left(3+\beta^{2}+2 \eta^{2}\right) \\
& -\chi_{2} \beta\left(1-\eta^{2}\right)\left(1+2 \beta^{2} \eta^{2}+3 \beta^{2}\right)=0,
\end{aligned}
$$

and

$$
\begin{aligned}
& 4 \beta \eta\left(1+\beta^{2}\right)-2\left(1+\beta^{2}\right)^{2}-\chi_{1} \eta\left(1-\eta^{2}\right)\left(3+2 \beta^{2}+3 \eta^{2}\right) \\
& -\chi_{2} \eta\left(1-\eta^{2}\right)\left(2 \beta^{2}+3 \beta^{4} \eta^{2}+3 \beta^{4}\right)=0 .
\end{aligned}
$$

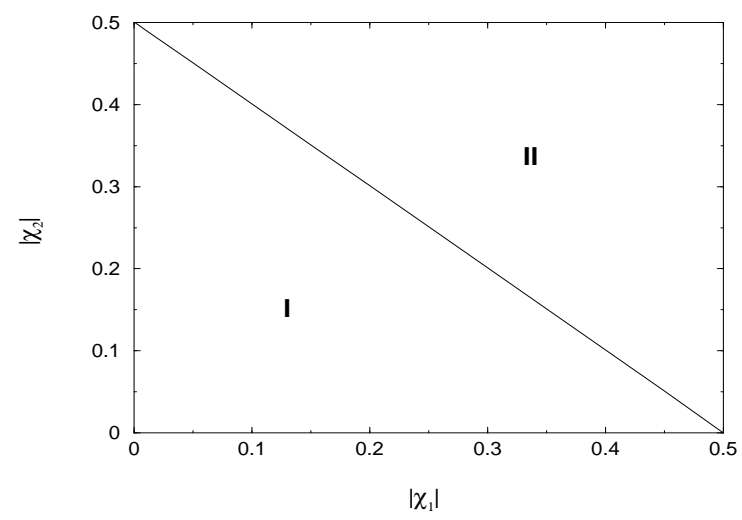

FIG. 2. Phase diagram of asymmetric SL states for the one-dimensional chain with a asymmetric-dimer impurity. Solid line is the critical line separating no-asymmetric-state region (marked as I) from the one-asymmetric-state region (marked as II).

Plotted in Fig. 2 is the phase diagram obtained from the solutions of Eqs. (15) and (16). There is only one critical line (for $\left.\left|\chi_{1}\right| \neq\left|\chi_{2}\right|\right)$ separating the no-asymmetric-state region (marked as I) from the one-asymmetric-state region (marked as II). The critical line in Fig. 2, in fact, turns out to be very closed to the solid critical line in Fig. 1. However, $\left|\chi_{1}\right|=\left|\chi_{2}\right|$ is a special case as far as asymmetric SL state is concerned. For $\left|\chi_{1}\right|=\left|\chi_{2}\right|$, the critical value of $|\chi|$ separating the no-state from the onestate region is 2.015. The full phase diagram of SL states for the dimer impurity may be obtained by superposing Fig. 1 and Fig. 2 (not shown). It is clear from the Fig. 1 and Fig. 2 that there are three distinct regions having one, two, and four states in the $\left(\left|\chi_{1}\right|,\left|\chi_{2}\right|\right)$ plane. Furthermore we see that there is a continuity as we go 
from one region to another. For the symmetric dimer $\left(\left|\chi_{1}\right|=\left|\chi_{2}\right|=|\chi|\right)$, we obtain three critical points, i.e., $\left|\chi_{c r}^{(1)}\right|=0.25,\left|\chi_{c r}^{(2)}\right|=2.015$ and $\left|\chi_{c r}^{(3)}\right|=2.732$. There is no SL state for $|\chi|<\left|\chi_{c r}^{(1)}\right|$, one state for $\left|\chi_{c r}^{(1)}\right| \leq|\chi|<$ $\left|\chi_{c r}^{(2)}\right|$, two states for $\left|\chi_{c r}^{(2)}\right| \leq|\chi|<\left|\chi_{c r}^{(3)}\right|$, three states for $|\chi|=\left|\chi_{c r}^{(3)}\right|$ and four states for $|\chi|>\left|\chi_{c r}^{(3)}\right|$. The corresponding critical values obtained by Eq. (1) are 1, $8 / 3$ and 8 respectively. Thus, in case of symmetric dimer also it is observed that the nearest neighbor coupling of the lattice oscillators is responsible to reduce the critical values, while the maximum number of SL states remain unchanged. However, it should be noted that the asymmetric nature of the dimer does play a crucial role to reduce the critical value of nonlinear strength abruptly.

\section{FULLY NONLINEAR CHAIN}

We now consider a periodically modulated nonlinear chain, i.e., the alternate sites are of the same nonlinear strength but any two consecutive sites are of the different strength. The distribution of the nonlinear strength, $\chi_{n}$ is given by

$$
\chi_{2 n+1}=\chi_{1} ; \quad \chi_{2 n}=\chi_{2} ; n=-\infty, \ldots-1,0,1, \ldots \infty .
$$

When $\chi_{1}=\chi_{2}$, the system reduces to a perfectly nonlinear chain. In both cases the translational symmetry of the system remains preserved. The Hamiltonian for this system is given by Eq. (3) with $\chi_{n}$ as defined in Eq. (17). Using the stationarity condition, $C_{n}=\phi_{n} \exp (-i E t)$, we can obtain the Hamiltonian in terms of $\phi_{n}$. In this case it is not possible to find the exact ansatz for the localized states, but there are a few rational choices. For example, the on-site peaked as well as the inter-site peaked and dipped solutions are possible. We will consider these cases subsequently.

\section{A. On-site peaked solution}

Since the lattice is periodically modulated with a period of two, the localized solution may have peak either at a site having nonlinear strength $\chi_{1}$ or at a site having strength $\chi_{2}$. We will carry out the calculation assuming that the solution is peaked at the center site (site 0 ). The result for the solution peaked at first site (site 1) may easily be obtained by interchanging the two nonlinear strengths in the resulting expression for the case of the center site peaked solution. Therefore, using the ansatz $\phi_{n}=\phi_{0} \eta^{|n|}$ and the normalization condition we get the effective Hamiltonian,

$H_{\mathrm{eff}}=\frac{4 \eta}{1+\eta^{2}}-\frac{\chi_{2}}{2}\left(\frac{2 \eta^{2}\left(1-\eta^{2}\right)}{\left(1+\eta^{2}\right)^{3}}+\frac{2\left(1-\eta^{2}\right)\left(1+\eta^{8}\right)}{\left(1+\eta^{2}\right)^{3}\left(1+\eta^{4}\right)}\right)$

$$
-\frac{\chi_{1}}{2}\left(\frac{2 \eta^{2}\left(1-\eta^{2}\right)}{\left(1+\eta^{2}\right)^{3}}+\frac{4 \eta^{4}\left(1-\eta^{2}\right)}{\left(1+\eta^{2}\right)^{3}\left(1+\eta^{4}\right)}\right) .
$$

After analyzing the fixed point equation, $\partial H_{\text {eff }} / \partial \eta=0$, we find that there is only one SL state. Interchanging $\chi_{1}$ with $\chi_{2}$, we obtain another SL state. Therefore for fixed value of $\chi_{1}$ and $\chi_{2}$, we will have two SL states, one peaked at a site of nonlinear strength $\chi_{1}$ and another peaked at a site of nonlinear strength $\chi_{2}$. Thus we conclude that if the nonlinear chain is modulated with periodicity $n$, there will be $n$ number of on-site peaked localized solutions. We note that the extra SL state due to extra periodicity in the periodically modulated chain was overlooked when the same system was studied by the use of Eq. (1) 14. These two states get closer and become the same as $\chi_{1} \rightarrow \chi_{2}$. After substituting $\chi_{1}=\chi_{2}=\chi$ in the fixed point equation we obtain

$$
\frac{1}{|\chi|}=\frac{\eta}{1-\eta^{2}}
$$

Equation (19) clearly indicates that there is one on-site site peaked solution (SL state) for a perfectly nonlinear chain, irrespective of the strength of the nonlinearity.

\section{B. Inter-site peaked and dipped solution}

For the inter-site peaked and dipped solutions we use the dimer ansatz in Eq. (9). After some algebra we obtain the effective Hamiltonian for the reduced dynamical system as

$$
\begin{array}{r}
H_{\mathrm{eff}}=2 \operatorname{sgn}(E) \eta+\frac{2 \beta\left(1-\eta^{2}\right)}{\left(1+\beta^{2}\right)}-\tilde{\chi}\left(\frac{\beta^{2}(1-\eta)^{2}}{\left(1+\beta^{2}\right)^{2}}\right) \\
+\widetilde{\chi}\left(\frac{\eta^{6}\left(1-\eta^{2}\right)^{2}\left(1+\beta^{4}\right)}{\left(1+\beta^{2}\right)^{2}\left(1-\eta^{8}\right)}+\frac{\eta^{2}\left(1-\eta^{2}\right)^{2}\left(1+\beta^{4}\right)}{\left(1+\beta^{2}\right)^{2}\left(1-\eta^{8}\right)}\right) \\
-\frac{\chi_{1}}{2}\left(\frac{2\left(1-\eta^{2}\right)^{2}\left(1+\beta^{4} \eta^{4}\right)}{\left(1+\beta^{2}\right)^{2}\left(1-\eta^{8}\right)}\right) \\
-\frac{\chi_{2}}{2}\left(\frac{2\left(1-\eta^{2}\right)^{2}\left(\beta^{4}+\eta^{4}\right)}{\left(1+\beta^{2}\right)^{2}\left(1-\eta^{8}\right)}\right)
\end{array}
$$

where $\tilde{\chi}=\chi_{1}+\chi_{2}$. We first consider the case, $\beta= \pm 1$. Substituting $\beta= \pm 1$ to the Eq. (20) we obtain

$$
\frac{4}{\left|\widetilde{\chi}^{ \pm}\right|}=\frac{\eta\left(2+\eta^{2}-\eta^{4}\right)}{(1 \mp \eta)\left(1+\eta^{2}\right)}
$$

from the fixed point equation. Here the ' + ' corresponds to the symmetric SL states and the '-' to the antisymmetric SL states. From Eq. (21) it is obvious that there will always be one symmetric solution for any point in the $\left(\left|\chi_{1}\right|,\left|\chi_{2}\right|\right)$ plane. But for antisymmetric solutions there are two critical lines. One being $\left|\chi_{2}\right|=6.427-\left|\chi_{1}\right|$ ( dotted line in Fig. 3) and another $\left|\chi_{2}\right|=8-\left|\chi_{1}\right|$ (solid line in Fig. 3). Three distinct regions (in Fig. 3) separated by the critical lines are marked by I, II and III. Region I has 
no SL state, region II has two antisymmetric states and region III has only one antisymmetric state. Furthermore we note that, for $\chi_{1}=\chi_{2}=\chi$, there is no antisymmetric state for $|\chi|<3.2135$, two states for $3.2135<|\chi| \leq 4$, and one solution for $|\chi|>4$. These critical values are again lower than those obtained from Eq. (1).

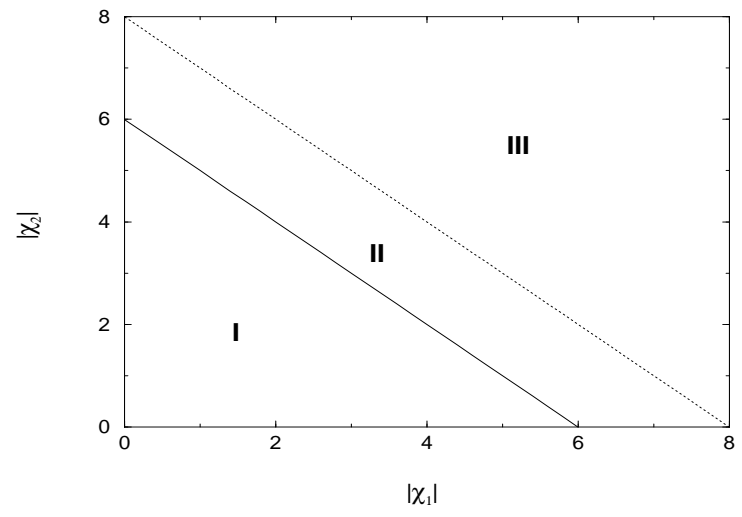

FIG. 3. Phase diagram of SL states for a periodically modulated nonlinear chain. The regions I, II and III has no, two and one antisymmetric SL state respectively.

Now, we look for the asymmetric solution corresponding to $\beta \neq 1$. The fixed-point equations are lengthy and, therefore, are not presented here. The fixed-point equations are solved numerically and the possible number of asymmetric SL states in the $\left(\left|\chi_{1}\right|,\left|\chi_{2}\right|\right)$ plane are found. It turns out that there is always one asymmetric SL state in the $\left(\left|\chi_{1}\right|,\left|\chi_{2}\right|\right)$ plane except along the line $\left|\chi_{1}\right|=\left|\chi_{2}\right|$ on which there exists a critical point, $\left|\chi_{1}\right|=\left|\chi_{2}\right|=1.75$, below which there is no asymmetric SL state and above which one asymmetric SL state. Thus, it is found that the modulation of nonlinearity reduces the critical value to obtain a localized state.

We emphasize that the SL states obtained in the fully nonlinear systems are based on the rational ansatz. However, many other SL states may possibly exist in the system. Thus, we are not in a situation to conclude about the maximum number of SL states for the fully nonlinear chain.

\section{RANDOM NONLINEARITY}

Here we consider a fully nonlinear chain on which all sites have random nonlinear strengths. It is known that, due to static random site energies, all the states in the system are localized and the system behaves as an insulator. The question is whether or not all the states are localized due to random nonlinearities in the system. Since the appropriate ansatz is not known, we calculate the mean square displacement of a particle in the system consisting of random nonlinear site energies [19]. The evolution of exciton is defined by Eq. (2) with $\chi_{n}$ being chosen randomly with equal probability for all $n$. The mean square displacement is defined as

$$
m(t)=\sum_{i=-\infty}^{\infty} i^{2}\left|C_{i}(t)\right|^{2} .
$$

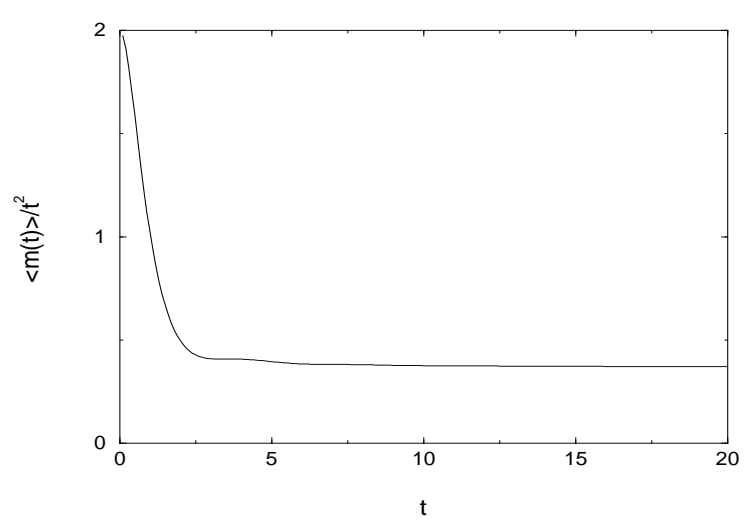

FIG. 4. The $<m(t)>/ t^{2}$ is plotted as a function of time $t$.

Since the set of coupled equations [Eq. (2)] can not be solved analytically, we solve them numerically using the fourth order Runge-Kutta method. Self-expanding lattice is used to avoid the finite size effect. The particle is initially placed at site 0 i.e., the center site. The time interval for the calculation is taken to be 0.001 . The total probability of the particle in the system should be conserved and it has been confirmed at every time step of our simulation. The mean square displacement is averaged over hundred random configurations and denoted as $<m(t)>$. Shown in Fig. 4 is $<m(t)>/ t^{2}$ as a function of $t$. We see that $\left\langle m(t)>/ t^{2}\right.$ initially decreases and becomes constant, much smaller than 2 . Thus, we conclude that there is a partial ballistic flow of the particle and hence all the states are not localized unlike the case of a linear system with random site energies.

\section{STABILITY}

The stability of the SL states can be understood from a flow diagram. For this purpose, we consider antisymmetric solutions due to the presence of a symmetric dimer in one dimensional chain. The reduced dynamical Hamiltonian for this case is given by

$$
H_{\mathrm{eff}}=-\left(1-\eta^{2}\right)+2 \eta+\frac{|\chi|}{4}\left(\left(3+\eta^{2}\right)\left(1-\eta^{2}\right)^{2}\right)
$$

The flow diagram of the dynamical system described by the $H_{\text {eff }}$ is constructed in the following manner. We treat 
$\partial H_{\text {eff }} / \partial \eta=f(\eta)$ as the velocity and $\eta$ as the coordinate of the dynamical system. We fix $\chi$ to be 3 for which two SL states appear. $f(\eta)$ is plotted in Fig. 5 as a function of $\eta$. A and B are the fixed points corresponding to the SL states. If $f(\eta)>0$, the flow of the dynamical variable is in the right direction, or else, in the left direction, as indicated by arrows in the figure. It is clear from the flow diagram that A is a stable fixed point, whereas B is unstable. Therefore, of the two antisymmetric SL states one is stable and another one is unstable. Similarly, we analyze the stability for all the states. It is found that if $N$ states exist for a particular case, $N / 2$ states are stable provided that $N$ is even and, otherwise $(N+1) / 2$ states are stable.



FIG. 5. The flow diagram where $f(\eta)$ is plotted as a function of $\eta$. A and $\mathrm{B}$ are the fixed points. Point $\mathrm{A}$ is a stable while $\mathrm{B}$ is unstable.

\section{CONCLUSION}

Modified discrete nonlinear Schrödinger equation has been used to study the formation of stationary localized states in a one-dimensional system with a single nonlinear impurity and a dimer (symmetric as well as asymmetric) nonlinear impurity. It is found that the critical values of nonlinear strength decrease due to the nearest neighbor coupling of the lattice oscillators. Furthermore, a periodically modulated nonlinear chain is studied and new SL states are found due to the modulation of nonlinearity. It is important to note that there will be more number of SL states if the periodicity of the modulation is larger. Another interesting point to note is that the critical value of nonlinearity to obtain SL state decreases drastically due to the modulation of the nonlinearity in a fully nonlinear chain and the asymmetric nature of the dimer impurity. The phase diagrams of SL states are presented for all the cases. The mean square displacement of a particle in one-dimensional chain with random nonlinear site energies is calculated to investigate whether all the states are localized as it happens in the case of a linear system. However, it is found that a fraction of the particle flows ballistically indicating that all the states are not localized even in the presence of random nonlinearities through out the system. It is further found that SL states may appear even in a perfectly nonlinear system. Thus, one may call these SL states as self-localized states. The stability of the SL states is also discussed. To see the effect of these nonlinearities on the formation of SL states in lattices with higher connectivity and higher dimension, the Cayley tree may be a good candidate as a linear host lattice.

[1] E. N. Economou, Green's Function in Quantum Physics (Springer-Verlag, Berlin. 1979).

[2] J. C. Eilbeck, P. S. Lomdhal and A. C. Scott, Physica D 16318 (1985).

[3] M. I. Molina and G. P. Tsironis, Phys. Rev. B 4715330 (1993).

[4] M. I. Molina, G. P. Tsironis and D. Hennig, Phys. Rev. E 502365 (1994).

[5] M. I. Molina and G. P. Tsironis, Int. Jour. Mod. Phys. B 91899 (1995).

[6] Y. Y. Yiu, K. M. Ng and P. M. Hui, Phys. Lett. A 200 325 (1995); Solid State Commun. 95801 (1995).

[7] P. M. Hui, Y. F. Woo and W. Deng, J. Phys. Condens. Matt. 82011 (1996).

[8] A. B. Aceves et al., Phys. Rev. E 531172 (1996).

[9] B. Melomed and M. I. Weinstein, Phys. Lett. A 22091 (1996).

[10] B. C. Gupta and K. Kundu, Phys. Rev B 55894 (1997).

[11] B. C. Gupta and K. Kundu, Phys. Rev B 5511033 (1997).

[12] K. Kundu and B. C. Gupta, Euro. Phys. Jour. B 3 (1998).

[13] B. C. Gupta and K. Kundu, Phys. Lett. A 235176 (1997).

[14] A. Ghosh, B. C. Gupta and K. Kundu, J. Phys. Conden. Matt. 102701 (1998).

[15] V. M. Kenkre, G. P. Tsironis and D. K. Campbell, Nonlinearity in Condensed Matter, eds. A. R. Bishop et al. (Springer-Verlag, 1987).

[16] V. M. Kenkre and G. P. Tsironis, Phys. Rev. B 351473 (1987).

[17] G. Kopidakis, C. M. Soukoulis, and E. N. Economou, Phys. Rev. B 497036 (1994); 51, 15038 (1995).

[18] Bikash Chandra Gupta, Phys. Rev. B 60, 6194 (1999).

[19] M. I. Molina and G. P. Tsironis, Phys. Rev. Lett. 73464 (1994). 